Efficient quantum computing with weak measurements

This article has been downloaded from IOPscience. Please scroll down to see the full text article.

2011 New J. Phys. 13053024

(http://iopscience.iop.org/1367-2630/13/5/053024)

View the table of contents for this issue, or go to the journal homepage for more

Download details:

IP Address: 132.234.251.222

The article was downloaded on 04/08/2011 at 23:42

Please note that terms and conditions apply. 


\title{
Efficient quantum computing with weak measurements
}

\author{
A P Lund \\ Brisbane, Queensland 4111, Australia \\ E-mail: a.lund@griffith.edu.au \\ New Journal of Physics 13 (2011) 053024 (16pp) \\ Received 25 January 2011 \\ Published 16 May 2011 \\ Online at http://www.njp.org/ \\ doi:10.1088/1367-2630/13/5/053024
}

Centre for Quantum Computation and Communication Technology (Australian Research Council), Centre for Quantum Dynamics, Griffith University,

\begin{abstract}
Projective measurements with high quantum efficiency are often assumed to be required for efficient circuit-based quantum computing. We argue that this is not the case and show that the fact that they are not required was actually known previously but was not deeply explored. We examine this issue by giving an example of how to perform the quantum-ordering-finding algorithm efficiently using non-local weak measurements considering that the measurements used are of bounded weakness and some fixed but arbitrary probability of success less than unity is required. We also show that it is possible to perform the same computation with only local weak measurements, but this must necessarily introduce an exponential overhead.
\end{abstract}




\section{Contents}

1. Introduction 2

2. Weak measurements 3

2.1. Projector probability observables . . . . . . . . . . . . . . 4

2.2. The single-qubit measurement model . . . . . . . . . . . . . . 4

2.3. Multi-qubit measurements . . . . . . . . . . . . . . . 6

2.4. Measuring probabilities in the computational basis . . . . . . . . 6

3. Algorithms with weak measurements $\quad 7$

3.1. Algorithmic complexity . . . . . . . . . . . . . . . . 7

3.2. Satisfiability with expectation values . . . . . . . . . . . 7

3.3. Order finding with expectation values . . . . . . . . . . . 8

4. Discussion 13

4.1. Local weak measurements . . . . . . . . . . . . . . . . . 13

4.2. Decoherence times . . . . . . . . . . . . . . . . . . . . . 14

4.3. Fault tolerance . . . . . . . . . . . . . . . . . . . 15

4.4. Implications for the experimental implementation of quantum computing . . . 15

5. Conclusion $\quad 15$

Acknowledgments $\quad 15$

$\begin{array}{ll}\text { References } & 15\end{array}$

\section{Introduction}

The work of DiVincenzo [1] states explicit requirements for scalable circuit-based quantum computing. Given the current state of the art, meeting these requirements in even moderately sized systems is technologically challenging ([2] and references therein). With some more modern implementations the criteria can be difficult to apply, but a certain reinterpreted set of criteria will apply for any particular implementation [2]. DiVincenzo's requirements consist of five criteria: well-defined scalable qubits, the ability to prepare fiducial states, near-perfect (below fault tolerant threshold) unitary evolution, access to a universal set of unitary evolutions and near-perfect quantum measurement.

There exists an assumption that the measurement criteria require strong projective measurements with near unit quantum efficiency to achieve the efficiency possible in quantum computing [3]. This may seem reasonable considering that the proposed quantum algorithms, which are efficient compared with the best-known classical algorithms, are presented with measurements in the basis of the eigenstates of Hermitian operators. Furthermore, models of quantum computing such as cluster state quantum computing [4] rely on strong measurements to perform the state transformations required for achieving universal computation. However, as DiVincenzo mentions [1], this is not a strict requirement and one can make trade-offs between conditions in order to achieve scalable quantum computing. The important issue is whether, when making a trade-off, algorithmic efficiency is lost.

This work was motivated by this brief observation of DiVincenzo to explicitly show a non-trivial example of an efficient quantum algorithm that involves non-ideal and, in particular, weak quantum measurements [5]. As a result, we hope to demonstrate in theory that, when building a demonstration quantum computer based on the circuit model, a strong projective 
measurement for readout in the computational basis is not absolutely necessary. This is an important consideration when constructing small- to medium-scale quantum computers as it allows for an extra degree of freedom, which can assist in the the design of algorithms matching the strength of the particular architecture used.

In this paper, we consider working in an architecture that is constrained in such a way that the interaction strengths for the readout measurements will only vary over a very small range and the time taken for the measurement is limited to small values to minimize the effects of decoherence. Within this constraint there has been some work to speed-up the measurement process by adapting the parameters as the measurement proceeds [6]. Here, we consider working in a non-adaptive regime and allow for arbitrarily small (but bounded) measurement strengths. As the information gained in each measurement is small the results from any algorithm must necessarily be formed by processing over an ensemble. The situation we consider is distinct from the situation found in bulk ensemble nuclear magnetic resonance quantum computing [7], as we will still require the preparation of pure quantum states before the computation.

This paper is organized as follows. In section 2, we describe weak measurements following the standard presentation given in the recent literature. Then we review a specific type of weak measurement on qubits, which differs slightly from the standard presentation but would be useful for our purposes. In section 3, we describe how to use these weak measurements in quantum computing, and give two specific examples of algorithms that may utilize such measurements. The two examples are of the satisfiability and order finding algorithms that, as we will show, lead to respectively inefficient and efficient use of weak measurements in quantum algorithms. In the penultimate section, we discuss the potential use of fault-tolerant constructions within this model and how using local weak measurements generally results in an inefficient overhead. Finally, we present the conclusions drawn from our results.

\section{Weak measurements}

Aharonov, Albert and Vaidman (AAV) [5] show how one can make a 'weak' measurement of an observable $A$ in which any single measurement outcome from the apparatus has very little information about the value of $A$ and is hence very noisy. However, averaging over a large enough ensemble, this noise can be removed and averages of $A$ can be recovered. It is possible to construct the measurement such that the lower the amount of information gathered about $A$, the less the system is disturbed. Quantum mechanics allows this disturbance to go to zero as the information obtained about $A$ goes to zero [8]. However, as the measurement becomes weaker, larger ensembles are required to mitigate the effects of noise and maintain a desired precision for the average of $A$.

AAV consider a measurement model with a system Hilbert space and a separate apparatus Hilbert space that describes the measuring apparatus. The apparatus space is assumed to have the same structure as a harmonic oscillator and the observable $X$ will represent the measurement outcomes and $P_{x}$ will be the generator of infinitesimal translations in $X$. The apparatus is also assumed to be in an initial state that is Gaussian and separable from the system. The system and apparatus are coupled by a Hamiltonian $H=g A \otimes P_{x}$, where $A$ is the observable that we wish to weakly measure and $g$ is a scalar value that will be a factor in determining the strength of the measurement. The observable $A$ can be any observable on the system. 
A system that is strongly isolated will have small values for the coupling constants in the Hamiltonian.

In the Heisenberg picture, the apparatus observable $X$ evolves to $X+g t A$, where $t$ is the interaction time for the coupling between the system and the apparatus. Knowing the strength and duration of the coupling and the initial state of the apparatus gives sufficient information for the statistics of $A$ to be calculated from the measurement results from the apparatus alone, irrespective of the strengths of the interaction. However, weaker measurements will require more measurements if some bound on the uncertainty in the statistical estimators needs to be achieved.

\subsection{Projector probability observables}

Projection operators are valid observables. The expectation value of such a projector observable is the modulus squared length of the component of the state within the subspace of the projector. In other words, if the projector is constructed from the space spanned by eigenstates of an observable with a particular eigenvalue, then the expectation value of the projector is the same as the probability that a strong measurement of the observable would result in that eigenvalue had it been made on the same ensemble. This idea of projectors as probability operators follows naturally from the generalized theory of quantum measurement.

If one can make a weak measurement of this projector observable, then it is possible to obtain this probability without actually having to actually perform the strong measurement of the underlying observable or greatly disturbing the system.

Finding a system with a Hamiltonian of the right form for a projector observable might be difficult, but one can use the quantum computing circuit model to construct a device that does with both the system and apparatus being qubits [9]-[11]. This construction is not the same as that considered in AAV, but of the same flavour. We will now describe this construction of a qubit weak measurement of a projector observable.

\subsection{The single-qubit measurement model}

Consider a measurement with both the system and apparatus Hilbert spaces being a single qubit. The system is assumed to be prepared in an arbitrary state $|\Psi\rangle$ and the apparatus is prepared in a pure state $\cos \theta|0\rangle+\sin \theta|1\rangle$ uncorrelated with the system (i.e. a separable state). Instead of specifying a Hamiltonian we will specify the coupling of the system and apparatus by a unitary gate, in particular the controlled NOT (CNOT) operation. Finally, the apparatus will be observed with the $Z=|0\rangle\langle 0|-| 1\rangle\langle 1|$ observable. This configuration is depicted in black in figure 1 .

If the $Z$ measurement of the apparatus is propagated back through the CNOT (cf the Heisenberg picture), then the final measurement of the apparatus is equivalent to a measurement of $Z \otimes Z$ on the system and the apparatus before the interaction. In other words, the measurement is equivalent to a measurement of the parity subspace on the combined input state. If the system state is written as $|\Psi\rangle=\alpha|0\rangle+\beta|1\rangle$, then the probabilities for the two measurement results will be

$$
\begin{aligned}
& P(+)=|\alpha|^{2} \cos ^{2} \theta+|\beta|^{2} \sin ^{2} \theta, \\
& P(-)=|\alpha|^{2} \sin ^{2} \theta+|\beta|^{2} \cos ^{2} \theta .
\end{aligned}
$$




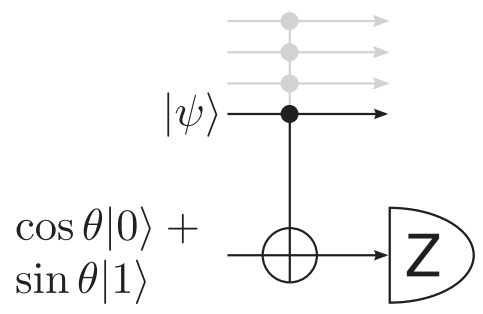

Figure 1. Model of a qubit-based weak measurement. An apparatus (the lowest shown qubit) is prepared in the state $\cos \theta|0\rangle+\sin \theta|1\rangle$ and measured in the $Z$ basis after interacting with the system (the upper qubits) prepared in an arbitrary state $|\psi\rangle$. The black lines show the case of a single-qubit weak measurement in which the interaction between the system and the meter is of the form of a singly-controlled NOT gate. The black and grey lines combined show the general many-qubit case of a multi-controlled NOT gate. The weakness of the measurement is determined by the parameter $\theta$, where when $\cos \theta=1$ the measurement is strong and equivalent to a projective measurement and when $\cos \theta=1 / \sqrt{2}$ the measurement is completely turned off. See the text for details.

From these equations it is possible to see that for $\theta=0$, the measurement output will be equivalent to a projective $Z$ measurement on the system. For $\theta=\pi / 4$, the output will give either result with equal probability independent of the system state. It is possible to show that when $\theta=\pi / 4$ the state of the system is undisturbed. This is unlike the AAV model in which the strength of the interaction is tuned not only from the initial meter state, but also by the strength of the interaction in the Hamiltonian and the interaction time. Here the full range of possible measurement strengths are achieved by tuning the initial meter state. However, one can think of this model as a $Z$ measurement on the system of AAV type.

The average value of $Z$ can be found from the expectation value of the function $\tilde{Z}$ defined by

$$
\begin{aligned}
& \tilde{Z}(x=+)=\frac{1}{\cos ^{2} \theta-\sin ^{2} \theta}, \\
& \tilde{Z}(x=-)=-\frac{1}{\cos ^{2} \theta-\sin ^{2} \theta},
\end{aligned}
$$

where $x$ is the meter measurement result. This function is well defined for $\theta \in[0, \pi / 4)$. The variance of this function on a single measurement is given by

$$
\frac{1}{\left(\cos ^{2} \theta-\sin ^{2} \theta\right)^{2}}-\left(|\alpha|^{2}-|\beta|^{2}\right)^{2}
$$

The variance can be understood as having a contribution of $\left(\cos ^{2} \theta-\sin ^{2} \theta\right)^{-2}-1$ from the variance due to the weakness of the measurement that can be infinitely large and $1-\left(|\alpha|^{2}-\right.$ $\left.|\beta|^{2}\right)^{2}$ from the variance of the system state that is at most 1 . For weak measurements the variance in the output is dominated by the variance due to the weakness of the measurement. This statement can be taken as a quantitative definition of measurement weakness. 
It is possible to make a measurement of the expectation value of the projector onto the +1 subspace of the $Z$ operator by the same apparatus but calculating the expectation value of the function

$$
\begin{aligned}
& \tilde{\Pi}_{Z}(x=+)=\frac{1}{2}+\frac{1}{2\left(\cos ^{2} \theta-\sin ^{2} \theta\right)}, \\
& \tilde{\Pi}_{Z}(x=-)=\frac{1}{2}-\frac{1}{2\left(\cos ^{2} \theta-\sin ^{2} \theta\right)},
\end{aligned}
$$

which has a mean of $|\alpha|^{2}$ for all $\theta$ values except $\pi / 4$ and a variance of

$$
\frac{1}{4\left(\cos ^{2} \theta-\sin ^{2} \theta\right)^{2}}-\frac{\left(|\alpha|^{2}-|\beta|^{2}\right)^{2}}{4} \text {. }
$$

A similar analysis of the contributions to the variance can be done as above.

\subsection{Multi-qubit measurements}

It is possible to extend this construction to build a larger class of weak measurements of projectors using multiply-controlled NOT gates. This configuration is depicted in the combined black and grey schematic in figure 1. Multiply-controlled NOT gates can be built efficiently using $O\left(n^{2}\right)$ singly-controlled gates and local unitaries [14]. A measurement of $Z$ on the meter after the interaction is equivalent to a measurement of the operator

$$
\begin{aligned}
& \hat{P}_{\perp} \otimes|0\rangle\langle 0|+| 111 \cdots\rangle\langle 111 \cdots|\otimes| 1\rangle\langle 1| \\
& -\hat{P}_{\perp} \otimes|1\rangle\langle 1|-| 111 \cdots\rangle\langle 111 \cdots|\otimes| 0\rangle\langle 0|
\end{aligned}
$$

on the system and meter Hilbert spaces before the interaction, where $\hat{P}_{\perp}$ is the projector onto the subspace that is the complement of the all ones subspace (i.e. the subspace that is spanned by all the qubit basis states except $|1111 \cdots 1\rangle$ ).

If the apparatus is prepared as in the case with a single control and the system is in the state $|\psi\rangle$, then the probabilities of the two outcomes of the apparatus measurement are

$$
\begin{aligned}
& P(+)=\left\langle\psi\left|\hat{P}_{\perp}\right| \psi\right\rangle \cos ^{2} \theta+|\langle 111 \cdots \mid \psi\rangle|^{2} \sin ^{2} \theta, \\
& P(-)=\left\langle\psi\left|\hat{P}_{\perp}\right| \psi\right\rangle \sin ^{2} \theta+|\langle 111 \cdots \mid \psi\rangle|^{2} \cos ^{2} \theta .
\end{aligned}
$$

This distribution is the same as that with the singly-controlled CNOT gate, but with the probabilities for the qubit in the system being in the one state replaced by the expectation values of the projectors onto the space with all ones. The mean and variances as calculated above also follow this replacement of variables. Therefore, the nature of the statistics does not change as the input size of the system Hilbert space increases.

\subsection{Measuring probabilities in the computational basis}

This model can also be used to measure the expectation value of projectors onto any one-dimensional subspace generated by a particular computational basis state by placing $X=|0\rangle\langle 1|+| 1\rangle\langle 0|$ gates before the measurement to transform the desired subspace into the all ones subspace. 
The value of the probability can be read out from the data collected at the meter by calculating the expectation value for the estimator of the average of the projection operator given above. Using the assumption of a weak measurement and large sample sizes, we can apply the central limit theorem to the estimator for the probability to calculate the uncertainty in the estimate of the expectation value. With some fixed error probability $\epsilon$ the estimate confidence interval is symmetric around the mean value and has width

$$
2 \sqrt{2} \frac{\sigma}{\sqrt{M}} \operatorname{erf}^{-1}(1-\epsilon) \leqslant \frac{\operatorname{erf}^{-1}(1-\epsilon)}{\sqrt{2 M}\left(\cos ^{2} \theta-\sin ^{2} \theta\right)},
$$

where $\sigma$ is the standard deviation of the measurement results, $M$ is the number of measurements made and erf is the standard error function.

Measuring the projectors spanned by multiple computational basis states can be simplified for some particular combinations of states. If the states contain all combinations of particular qubits with all other qubits constant, then the qubits that vary can simply be not measured. However, if even a single qubit combination is missing, then each combination must be measured separately.

\section{Algorithms with weak measurements}

In this section, we describe quantum computing algorithms in terms of the expectation values and decision problems, but analyse the complexity by restricting ourselves to the qubit weak measurement just described.

\subsection{Algorithmic complexity}

It is assumed that there is some (presumably small) fixed error tolerance allowed for the computation as a whole. For algorithms utilizing the weak qubit measurement readout just described, the temporal computational complexity is then determined by the number of repetitions required to achieve this error value. If under these conditions the quantum algorithm has a polynomial temporal complexity, it lies in the BQP complexity class (the class of practical quantum problems).

We are going to assume that the strength of all measurements is well known and greater than some fixed constant value. Hence, a worst-case value is known for the uncertainty in the output measurement statistics, and we will assume that this worst-case value is the actual estimate of the uncertainty. We are also going to assume that sample sizes are large enough for the central limit theorem to apply. We are not going to be dealing with any distributions in which the central limit theorem is not valid. These assumptions combined allow the variance of the sample mean to be computed and hence a signal-to-noise ratio involving the estimated mean and the worse-case standard deviation can be used to infer the maximum probability of error inherent in the computation.

\subsection{Satisfiability with expectation values}

The satisfiability problem is defined as identifying whether a logical statement described by a Boolean function $f$ has a set of inputs that result in the function evaluating be to true. 
If the function represents a conjunction of logical statements (the inputs), then the statement (the output) is said to be satisfied by the particular combination of truth values used to achieve this output. This problem lies in the class of decision problems.

Cory et al [12] construct a quantum algorithm for solving the satisfiability problem. In that paper, they assumed that the standard model of quantum computing is enhanced by special measurements that can extract expectation values of observables for a single instance of a quantum state in an error- and noise-free way. Their work was motivated by the nuclear magnetic resonance quantum computing model, so this type of measurement involving ensemble averages is a natural consequence of the output signals from that type of computation. They then show that given an equal superposition of all possible logical inputs onto the function, of which there are $2^{n}$ possibilities, a unitary that implements $f$ can be built efficiently and evaluates all of these possibilities coherently in superposition. The unitary is built so that the output value of the function is written onto another qubit register, which is zero if the input does not satisfy the statement and one if it does. The expectation value of the output register is then obtained using the special measurement that they added as described above. If the expectation value is non-zero, then the logical statement is satisfiable. Although this does not say which input will satisfy the function, it does show that such a satisfying input exists. Provided one has this enhancement that allows for the immediate extraction of expectation values, this is a method of solving an NP-complete problem (i.e. satisfiability) deterministically in polynomial time.

This result is possible only when neglecting the noise in the output of such a measurement. If one requires this measurement to be a standard quantum measurement rather than the special one used, then the complexity will change as more measurements are required to counter the effects of the noise. Consider the possible case where only a single particular input satisfies the function (as is possible for any size satisfiability problem). A measurement that determines satisfiability then needs to distinguish between two cases, the case of being unsatisfiable and the output register is in the state $|0\rangle$ and the case of having a single satisfying input and the output register is in the state $\left(1-2^{-n}\right)|0\rangle\left\langle 0\left|+2^{-n}\right| 1\right\rangle\langle 1|$. The distinguishing property of these two states is the probability of measuring the output register in the state $|1\rangle$. Therefore, the readout must estimate a probability of size $2^{-n}$, and if the number of measurements made, $M$, is large, then, as the output is binomially distributed, the noise in the estimate of the probability approaches $2^{-n / 2} / \sqrt{M}$. Hence, the signal-to-noise ratio decreases exponentially in the size of the input. This overcomes the apparent speed-up offered by the enhanced model of quantum computing considered as the sample size needed to achieve a particular probability of error in the decision problem will increase exponentially. This overhead due to the nature of quantum measurements applies to all types of possible readout measurements, be they weak measurements or not.

As we show next, not all useful ensemble averages from the output of quantum computations necessarily have this problem.

\subsection{Order finding with expectation values}

The order finding problem is a critical part of the quantum prime factorization algorithm [13]. The definition of the order finding problem is: given positive integers $N$ and $x<N$, find the least positive integer $r$ such that $x^{r}=1(\bmod N)$. This problem is an instance of the hidden subgroup problem, which is a more general class of problems [14]. The problem of factoring integers can be reduced to this problem [14]. Currently, the best-known classical algorithms have exponential complexity. 


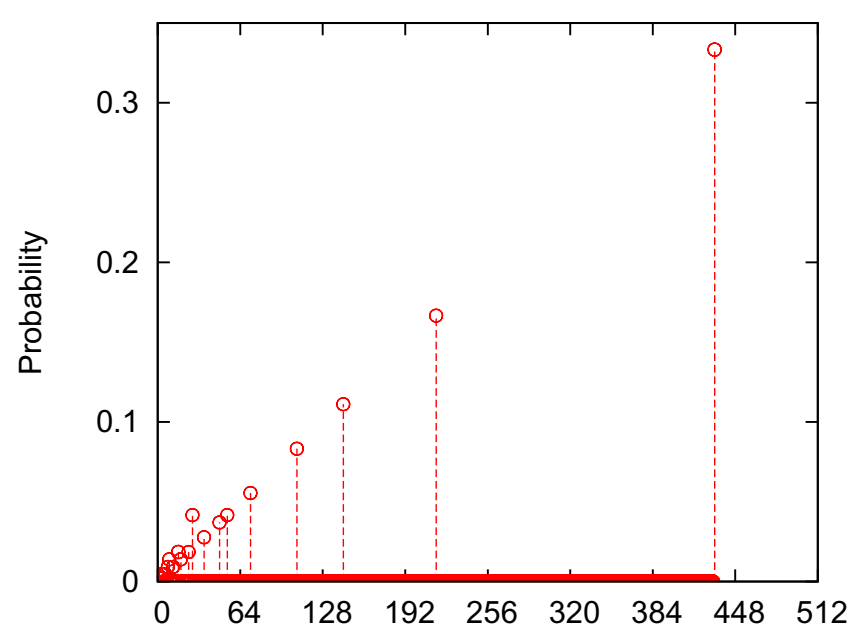

Figure 2. An example distribution of output values for the denominator register when the order is known to be 432 . If the order is prime, then all of the probability is concentrated at the highest value.

The quantum order finding algorithm utilizes a quantum modular exponentiation operation defined by the unitary

$$
U_{x, N}=|y\rangle=|x y(\bmod N)\rangle,
$$

which can be done efficiently using $O\left(n^{3}\right)$ gates where $n$ is the number of bits needed to represent integers up to $N$. The eigenvalues of this unitary are $\exp (2 \pi \mathrm{i} s / r)$, where $r$ is the order of $N$ and $s$ is an integer satisfying $0 \leqslant s<r$ which labels each of the eigenstates. Therefore, performing a quantum phase estimation on an eigenstate of the modular exponentiation operator is a method of finding information about the order of $x$ [14]. However, preparing the eigenstate would require that the order of the integer of interest be known already. Therefore, a superposition state of all possible eigenstates is used. This state happens to be equal to a state that is the representation of the multiplicative identity in the computational basis. Therefore, the output of the order finding algorithm is a phase $\phi=s / r$, where $r$ is the order that we desire and $s$ is equally distributed among the allowed values. The continued fractions expansion of $\phi$ allows for the computation of values for $r$. However, if $s$ and $r$ share a factor, then this method will give the value of $r$ with this factor divided out. This is then not the order that was desired but a factor of the order.

For a randomly selected value of $s$, the probability that it is prime for large values of $N$ is at least and will asymptotically approach

$$
\frac{1}{2 \log N} \geqslant \frac{1}{n(2 \log 2)} \text {. }
$$

This guarantees that there will be some probability that the correct answer is contained within the output and that this probability drops as $\Omega\left(n^{-1}\right)$ with the size of the problem. An example of the distribution for values of $r$ read out from the continued fractions algorithm is shown in figure 2 .

We are now in a position to describe the quantum order finding algorithm using only weak measurements. The procedure that will be described here is also shown schematically in 
(a)

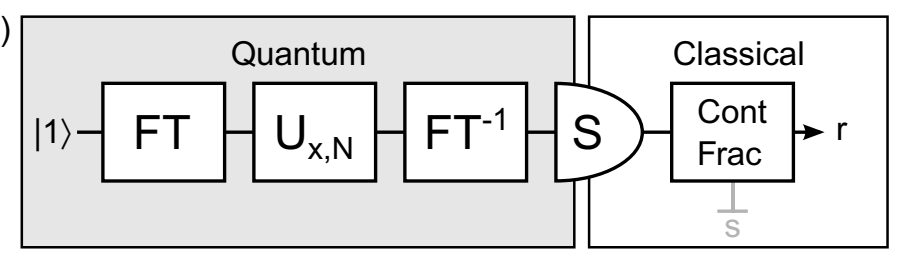

(b)

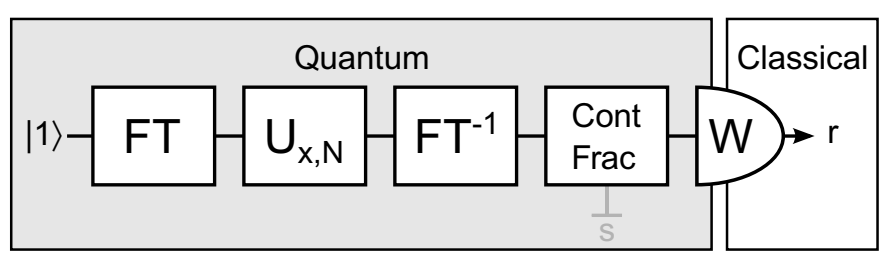

Figure 3. Schematic diagrams showing the difference between the strong (a) and weak (b) measurement versions of the ordering finding algorithm. FT represents the Fourier transform unitary and the unitary $U_{x, N}$ is defined in the text. S and $\mathrm{W}$ represent strong and weak measurement readout, respectively. The continued fraction algorithm as utilized for this order finding algorithm has two outputs, the numerator represented by $s$ and whose information is not utilized and the denominator register represented by $r$. They key distinction is that in this presentation the continued fractions algorithm is performed quantum mechanically in the weak measurement version.

figure 3. Firstly, build the order finding algorithm as done for projective readout measurement, but do not measure the register containing the phase $\phi=s / r$ result. This requires no projective measurements, only good state preparation and precise unitary evolution. Secondly, implement the continued fractions algorithm and calculate the rational convergent on the $s / r$ register in the computational basis quantum mechanically using a construction based on universal reversible gates [14]. This construction requires no measurement or feed-forward, but does require multiqubit conditional unitaries. This shifts $O\left(n^{3}\right)$ classical gates to quantum gates and represents part of the overhead to this method. Tracing over the numerator, the reduced density operator for the convergent's denominator register will be

$$
p|\Psi\rangle\langle\Psi|+(1-p) \hat{\rho}_{\mathrm{e}},
$$

where $|\Psi\rangle$ is the state of the denominator register representing $r$ (the result). $\hat{\rho}_{\mathrm{e}}$ is a density operator orthogonal to $|\Psi\rangle$ representing those terms when the numerator had a common factor with the denominator. The standard procedure for the readout is to make a strong projective measurement on this state in the computational basis to read out a result, test to see if it is a solution and repeat the algorithm (possibly modified) if the order is found not to be correct but a factor of the order. Here, we wish to only use weak measurements to extract the answer. It is clear that the largest value of any component from the denominator register will be the order we are seeking and not merely a factor of the order. Therefore, we propose to extract the register state with the largest numerical value through a bi-sectional search on the properties of denominator register using ensemble averages.

The bi-sectional search proceeds by a series of decision problems. The problems form the answer bit by bit, generating the largest value with non-zero probability from the most significant bit to the least significant bit. These probabilities are extracted by estimating expectation values via repeated measurements of carefully selected projectors on 
the denominator register ensemble as we will now detail. Consider the projector onto the space containing a logical one state for the most significant qubit of the denominator register and all other registers allowed any value. This projector is

$$
\hat{\Pi}_{1 \ldots}=\frac{1}{2}(Z-I) \otimes I \otimes I \otimes \ldots
$$

If the average of this projector was non-zero, then it is known that the largest numerical value in the computational basis for the denominator register state has its most significant bit as a 1 . If the average is zero, then the largest value must have a zero for the most significant bit. This procedure is this repeated for the next most significant bit using the appropriate projector adapted from the previous result. For example, if the most significant bit was a zero, then the next measurement to be made would be

$$
\hat{\Pi}_{01 \ldots}=\frac{1}{2}(Z+I) \otimes \frac{1}{2}(Z-I) \otimes I \otimes \ldots,
$$

or if it was a one, then the next measurement is

$$
\hat{\Pi}_{11 \ldots}=\frac{1}{2}(Z-I) \otimes \frac{1}{2}(Z-I) \otimes I \otimes \ldots
$$

This continues for each bit and when all have been read out the largest value with nonzero probability for the register is known. At each step, the projector representing the space containing the answer has its dimension halved; however, the size of the expectation value is bounded by the probability given in equation (14). An illustration of how the bi-sectional search works is shown in figure 4 .

To achieve an overall algorithmic error probability less than $\epsilon$, the error probabilities for each bit readout measurement must be less than $\epsilon / n$. This is because if the probability of failure for one run is $\epsilon^{\prime}$, then the overall probability of failure is $1-\left(1-\epsilon^{\prime}\right)^{n}<n \epsilon^{\prime}$ and hence $n \epsilon^{\prime}<\epsilon$. If we invoke the central limit theorem as foreshadowed above and assume that the estimator for the mean is normally distributed with a variance of $\sigma_{0}^{2} / n$ with $\sigma_{0}$ being the variance in a single outcome and we are deciding between two means of $s_{0}$ and $s_{1}$ (which we will call the signal), then we define $\mathrm{SNR}_{0}=\left|s_{1}-s_{0}\right| / \sigma_{0}$. Here we are assuming that the variance from the two distributions is equal. We can do this by choosing a worse-case variance as described above. Taking a threshold halfway between the two signal values, the probability of making an error is

$$
P_{\text {error }}=1-\Phi\left(\frac{\mathrm{SNR}_{0} \sqrt{M}}{2 \sqrt{2}}\right),
$$

where $\Phi$ is the cumulative distribution function of the standard normal distribution. We require this probability to be less than $\epsilon^{\prime}=\epsilon / n$. The number of samples required to meet the error budget must therefore satisfy

$$
M=\left[\frac{2 \sqrt{2}}{\mathrm{SNR}_{0}} \operatorname{erf}^{-1}\left(1-\frac{2 \epsilon}{n}\right)\right]^{2},
$$

which scales poly-logarithmically in $n$ for the bi-sectional search algorithm. To prove this scaling, rearranging this expression gives

$$
\operatorname{erf}\left(\frac{\mathrm{SNR}_{0} \sqrt{M}}{2 \sqrt{2}}\right)=1-\frac{2 \epsilon}{n},
$$


(a) ?\#\#\#\#\#\#\#

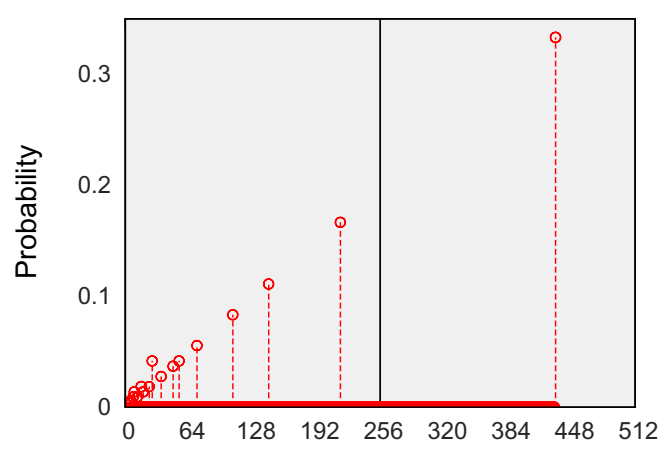

(c) 11?\#\#\#\#\#

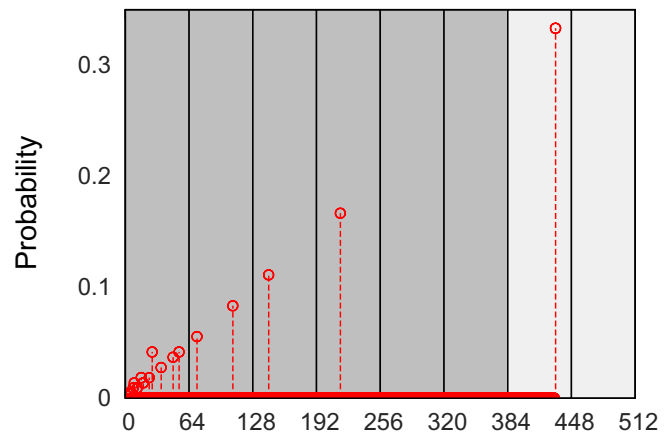

ФDEutsche PhysiKaLISCHE GeSELLSCHAFT

(b) 1?\#\#\#\#\#\#

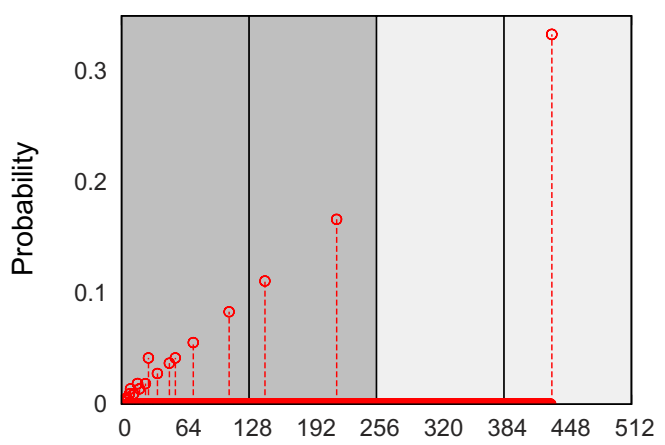

(d) 110?\#\#\#\#

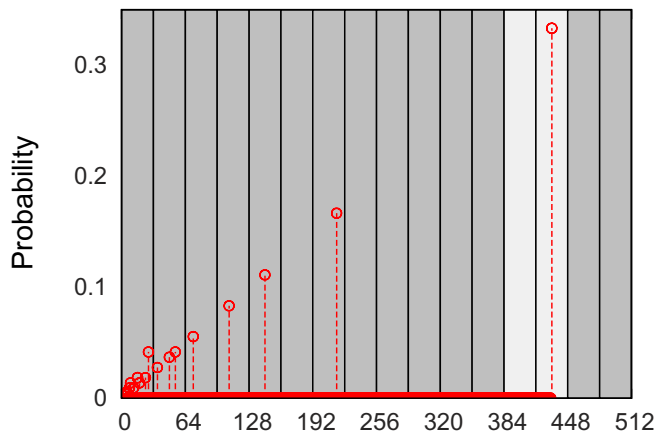

Figure 4. An illustration of the steps involved for the bi-sectional search for the readout of the largest value of the register with non-zero probability. The distribution chosen is the same as figure 2; hence, the answer read out should be a binary representation of 432 (110110000). Initially, the most significant bit is weakly measured, the remaining qubits are not measured and their values are not yet known. This state of knowledge is shown in the title of the plot of part (a) with \# representing unknown information that is not to be measured and ? representing unknown information that is currently being measured. The weak qubit measurement described in the text is used to determine whether there is any probability of the most significant bit value being one. The measurement is equivalent to determining whether there is any probability on the right-hand side of the plot as shown by the central dividing line. If there is any probability that the state is one (as is the case here), then the measurement proceeds to determining whether there is any probability in the output where the two most significant bits are $|11\rangle$, as depicted in part (b). If it was determined that there was no probability that the most significant bit was one, then the measurement proceeds to determining whether there is any probability in the output where the two most significant bits are $|01\rangle$. In either case, if there is a probability that the second most significant bit is one, then the state is kept and the search continues to the next most significant bit. If there is zero probability, then the bit being determined is flipped (e.g. $|11\rangle \rightarrow|10\rangle$ ) and the search then continues. This is repeated until all qubits are measured and the final readout value will be the largest non-zero probability value within the qubit register. If there are $n$ qubits, then this procedure has $n$ steps. 
which is equivalent to

$$
\frac{\epsilon}{n}=\frac{1}{\sqrt{\pi}} \int_{\mathrm{SNR}_{0} \sqrt{N} / 2 \sqrt{2}}^{\infty} \mathrm{e}^{-t^{2}} \mathrm{~d} t .
$$

For $M$ sufficiently large (in particular $\mathrm{SNR}_{0} \sqrt{M}>2 \sqrt{2}$ ),

$$
\begin{aligned}
\int_{\mathrm{SNR}_{0} \sqrt{M} / 2 \sqrt{2}}^{\infty} \mathrm{e}^{-t^{2}} \mathrm{~d} t & <\int_{\mathrm{SNR}_{0} \sqrt{M} / 2 \sqrt{2}}^{\infty} \mathrm{e}^{-t} \mathrm{~d} t \\
& =\mathrm{e}^{-\mathrm{SNR}_{0} \sqrt{M} / 2 \sqrt{2}} .
\end{aligned}
$$

Therefore,

$$
n>\epsilon \sqrt{\pi} \mathrm{e}^{\mathrm{SNR}_{0} \sqrt{M} / 2 \sqrt{2}}
$$

or rearranging

$$
\sqrt{M}<\frac{2 \sqrt{2}}{\mathrm{SNR}_{0}}(\log n-\log \epsilon \sqrt{\pi}),
$$

and therefore

$$
\left[\frac{2 \sqrt{2}}{\mathrm{SNR}_{0}} \operatorname{erf}^{-1}\left(1-\frac{2 \epsilon}{n}\right)\right]^{2}=O\left(n \log (n)^{2}\right),
$$

where we have used the $\Omega\left(n^{-1}\right)$ scaling of $\mathrm{SNR}_{0}$ from equation (14). Therefore, the number of total weak measurement samples needed in the algorithm is $\Omega\left(n \log (n)^{2}\right)$.

This requirement to make poly-logarithmically extra samples forms another part to the overhead of this procedure. Furthermore, the multiplying factor in the scaling will depend on the weakness of the measurement which may be large for very weak measurements. However, none of the overheads introduced in this presentation scale exponentially in the size of the input.

\section{Discussion}

\subsection{Local weak measurements}

Resch and Steinberg [15] have shown that it is possible to extract non-local weak values from local weak measurements. Therefore, one might be tempted to measure the multi-qubit expectation value using local single-qubit measurements instead of the non-local measurements used here. However, this does introduce an inefficient overhead.

In general, measuring an $n$-qubit output will need to have estimates of the expectation values for observables of the form $A_{1} A_{2} \cdots A_{n}$. When observing the correlations in the local meter readouts to estimate this value, the variance of the correlation constructed from all the meters is

$$
\operatorname{var}\left(X_{1} X_{2} \ldots X_{n}\right)_{\mathrm{o}}=\left\langle\left(X_{1} X_{2} \ldots X_{n}\right)^{2}\right\rangle_{\mathrm{o}}-\left\langle X_{1} X_{2} \ldots X_{n}\right\rangle_{\mathrm{o}}^{2}
$$

where $X$ represents the meter observables as per section 2 and the subscript o is a reminder that this description is for measurements at the meter output. If each meter is initialized separately 
with a mean of zero and a variance of $\sigma^{2}$, then the variance at the meter output in terms of the inputs becomes

$$
\begin{aligned}
\operatorname{var}\left(X_{1} X_{2} \cdots X_{n}\right)_{\mathrm{o}}= & \left\langle\left(X_{1}+\gamma A_{1}\right)^{2}\left(X_{2}+\gamma A_{2}\right)^{2} \cdots\left(X_{n}+\gamma A_{n}\right)^{2}\right\rangle_{\mathrm{i}}-\gamma^{2 n}\left\langle A_{1} A_{2} \cdots A_{n}\right\rangle_{\mathrm{i}}^{2} \\
& =\sigma^{2 n}+\gamma^{2} \sigma^{2(n-1)} \sum_{k=0}^{n}\left\langle A_{k}^{2}\right\rangle_{\mathrm{i}}+\gamma^{4} \sigma^{2(n-2)} \sum_{k=0}^{n} \sum_{l=0}^{n}\left\langle A_{k}^{2} A_{l}^{2}\right\rangle_{\mathrm{i}}+\cdots+\gamma^{2 n} \\
& \times \operatorname{var}\left(A_{1} A_{2} \ldots A_{n}\right),
\end{aligned}
$$

where we have used the commutativity of the different subsystems to rearrange terms and statistical independence of the meters and the meter and system to remove terms. This expression has a scaling of $O\left(\sigma^{2 n}\right)$ from the first term on the right-hand side which is independent of the actual signal from the system observable. Therefore, the $\mathrm{SNR}_{0}$ term decreases exponentially, introducing an inefficient overhead.

Observables of the type just mentioned are observed locally in the standard presentation of quantum computing algorithms using strong measurements. Clearly there must be a point of transition in the initial variance of the meter states compared to the measurement strength where the exponential scaling term from the meter noise does not play a significant role in the data extraction. This quantity will be dependent on the observables needed and hence the type of algorithm being implemented. For example, a fault-tolerant implementation would have a point in which the noise scaling decreases as the size of the observables rises, rather than increasing as is the case for this simple example.

Another possible way to avoid this problem of local weak measurement introducing an exponential overhead is to break the requirement of local preparation of initial meter states. If the initial meter state was correlated, then the equality reached above would change significantly. If the right state is chosen for the observable of interest, then it may be possible to avoid the exponential scaling.

Other works on non-local weak measurements in a completely different context have also found that estimating non-local correlations is inefficient and requires large ensembles [16, 17]. So it appears that for efficient quantum information processing with weak measurements, nonlocality is strictly required. This means that schemes for extracting conditional expectation values using informationally complete but not full strength measurements [20] cannot be used to perform efficient computation.

\subsection{Decoherence times}

One may argue that the weakness of the readout and the length of time for the output for the algorithm counteract one another. However, this is not true for the algorithm presented here as the algorithm is rerun, qubits are reprepared and the unitary evolution is run again, which removes any of the effects of previous decoherence. Hence, the important time to consider is decoherence over the time taken to execute all the operations needed to run the algorithm in total as is the case for strong measurements. With the standard model of weak measurements (as presented here and in [5]) the interaction time for a weak measurement is much smaller than that for the corresponding strong measurement and hence could act to reduce the effects of decoherence. 


\subsection{Fault tolerance}

Fault tolerance encoding, evolution and decoding can still be performed if the final measurements are not strong measurements. For the CSS class of quantum codes, one can avoid using measurements completely and still achieve fault tolerance [18]. Doing so involves some penalty in the fault-tolerant threshold, but as shown recently this penalty is not as great as has been believed previously [19].

\subsection{Implications for the experimental implementation of quantum computing}

This result suggests that in the pursuit of preliminary or proof-of-principle quantum computing experiments, strong-isolation and high-fidelity operations are where effort should focus provided one has the ability to read out data even if very noisy. For the order finding algorithm presented here, having a weak readout does not harm the efficiency of quantum computing. Increasing the strength of the readout clearly has an advantage in the rate at which computation can occur, but this should not be done to the detriment of the ability for the data to be preserved within the quantum computer to complete the computation.

\section{Conclusion}

We have outlined how weak measurements in quantum computing can be modelled theoretically and modified a quantum algorithm using this model in such a way that the computational efficiency of performing the algorithm quantum mechanically is maintained. The requirements on state preparation and control over the evolution are the same as for any other model of quantum computation. This may be able to assist in the technological challenge of building demonstration quantum computers.

\section{Acknowledgments}

APL acknowledges fruitful discussions with $\mathrm{H}$ M Wiseman and T C Ralph while preparing the manuscript. This research was conducted by the Australian Research Council Centre of Excellence for Quantum Computation and Communication Technology (project number CE110001027). This research was also supported by the Griffith University Postdoctoral Research Fellowship.

\section{References}

[1] DiVincenzo D P 2002 Fortschr. Phys. 48 771-84 (arXiv:quant-ph/0002077v3)

[2] Ladd T D, Jelezko F, Laflamme R, Nakamura Y, Monroe C and O’Brien J L 2010 Nature 464 45-53

[3] Morello A et al 2010 Nature 467 687-91

[4] Raussendorf R and Briegel H J 2001 Phys. Rev. Lett. 86 5188-91

[5] Aharonov Y, Albert D Z and Vaidman L 1988 Phys. Rev. Lett. 601351

[6] Combes J and Jacobs K 2006 Phys. Rev. Lett. 96010504

[7] Braunstein S L, Caves C M, Jozsa R, Linden N, Popescu S and Schack R 1999 Phys. Rev. Lett. 83 1054-7

[8] Ozawa M 2003 Phys. Rev. A 67042105

[9] Pryde G J, O’Brien J L, White A G, Ralph T C and Wiseman H M 2005 Phys. Rev. Lett. 94220405

[10] Ralph T C, Bartlett S D, O’Brien J L, Pryde G J and Wiseman H M 2006 Phys. Rev. A 73012113 
[11] Lund A P and Wiseman H M 2010 New J. Phys. 12093011

[12] Cory D G, Fahmy A F and Havel T F 1997 Proc. Natl Acad. Sci. USA 941634

[13] Shor P W 1997 SIAM J. Sci. Stat. Comput. 261484

[14] Nielsen M A and Chuang I L 2000 Quantum Computation and Quantum Information (Cambridge: Cambridge University Press)

[15] Resch K J and Steinberg A M 2004 Phys. Rev. Lett. 92130402

[16] Kedem Y and Viadman L 2010 Phys. Rev. Lett. 105230401

[17] Broduch A and Viadman L 2009 J. Phys.: Conf. Ser. 174012004

[18] Aharonov D and Ben-Or M 2008 SIAM J. Comput. 381207 (arXiv:quant-ph/9906129)

[19] Paz-Silva G A, Brennen G K and Twamley J 2010 Phys. Rev. Lett. 105100501

[20] Ralph T C, Huntington E H and Symul T 2008 Phys. Rev. A 77063817 